\title{
METAVERSOS FORMATIVOS. TECNOLOGÍAS Y ESTUDIOS DE CASO
}

Viviana Barneche-Naya1: Universidad de la Coruña. España viviana.barneche@udc.es

Rocío Mihura-López: Universidad de la Coruña. España $\underline{\text { mihura@udc.es }}$

Luis Antonio Hernández-Ibáñez: Universidad de la Coruña. España lhernandez@udc.es

\section{RESUMEN}

Este artículo analiza el grado actual de aplicación de los mundos virtuales 3D o Metaversos en el ámbito de la educación en los diferentes tramos formativos, recopilando ejemplos de aplicación llevados a cabo en todo el mundo y analizando las ventajas e inconvenientes de las distintas plataformas de creación y desarrollo de mundos virtuales disponibles para la comunidad educativa. Finalmente, describe varios casos de aplicación diseñados y desarrollados por el grupo de investigación de los autores, incidiendo incluso en aspectos relativos al diseño de ciberarquitectura que influyen en la adecuación de estas infraestructuras virtuales al fin al que se destinan.

PALABRAS CLAVE: Mundos Virtuales - Metaversos - E-learning - Ciberarquitectura - Código abierto 


\title{
EDUCATIONAL METAVERSES. TECHNOLOGY AND CASE STUDIES
}

\begin{abstract}
This paper analyzes the degree of application of the technology of virtual worlds, also called Metaverses in the field of education, gathering examples carried out throughout the world and describing the advantages and disadvantages of the different platforms that can be used for the creation and development of metaverses for educational purposes. Finally, it studies several cases developed by the authors' research group, detailing aspects relative to the design of ciberarchitecture, that have direct influence in the adequation of such virtual facilities to the use that they are intended.
\end{abstract}

KEY WORDS: Virtual Worlds - Metaverses - E-learning - Ciberarchitecture - Open source

\section{INTRODUCCIÓN}

Actualmente, el ciudadano global vive en constante interacción con los entornos virtuales surgidos de la web 2.0, donde participación y colaboración son la base de generación del conocimiento colectivo. En este contexto una de las tecnologías emergentes en los últimos años son los metaversos, mundos virtuales tridimensionales e inmersivos que permiten la interacción en tiempo real, la telepresencia y la creación de contenidos por parte de los usuarios.

Estos entornos virtuales, conocidos también como MUVE (Multi-UserVirtual Environments), poseen un formato que deriva de los MMORPG (Massive Multiplayer Online Role-Playing Games) aunque a diferencia de estos, no posen a priori un objetivo o meta a cumplir como sucede en un videojuego.

Diferentes estudios sobre el uso de las tecnologías prevén para los próximos años cinco años la utilización intensiva de los espacios tridimensionales virtuales (Telefónica I+D, 2009, Gartner's 2011 Hype Cycle) y un claro avance en los procesos de "gamification"."Gamification is an emerging business and societal trend with an extraordinarily broad reach across innovation. Gamification's potential to change human behavior makes it a trend to watch."(Gartner, 2011). 
La consultora Gartner vaticina que para el 2015 más del 50\% de las organizaciones que gestionan los procesos de innovación utilizarán los mecanismos de los juegos en procesos reales. Según esta investigación, el uso del juego en aplicaciones del mundo real puede aumentar drásticamente los niveles de interés del usuario ya que el uso de la interactividad y el reto provocan la motivación y el deseo de participar.

El análisis de diferentes fuentes y datos sobre esta tecnología (KZero Worldswide, 2011; NMC Horizon Report 2011- K12) ponen de manifiesto un panorama muy alentador en cuanto a sus alcances, potencialidades y grados de adopción por parte de los usuarios, en especial los "nativos digitales", quienes asumen de forma natural la utilización de los mundos virtuales como lugares de encuentro y de actividades con sus pares.

A partir de todos estos datos es indudable que los ambientes virtuales multiusuario presentan aspectos innovadores que constituyen un campo para la investigación, la experimentación y como uno de los grandes aportes en el uso de tecnología digital aplicada a la educación.

En este sentido empresas de investigación de mercado como Strategy Analytics ${ }^{2}$ han previsto que la población de mundos virtuales aumentará casi 3 veces y media hacia el 2015, debido en gran parte al uso de los mismos por parte de niños y adolescentes, quienes utilizan estos medios para interaccionar con otros usuarios/avatares en tiempo real, para comunicarse y realizar variadas actividades "juntos" en un mismo "lugar". El informe del New Media Consortium, "2011 Horizon Report" , prevé que para el año 2013 los mundos virtuales 3D colaborativos como como Second Life y OpenSimulator estarán presentes como una herramienta habitual en la educación secundaria:

Proponents of games-based learning in higher education point to its role in supporting collaboration, problem-solving, and communication [...] Advocates also underscore the productive role of play, which allows for experimentation, the exploration of identities, and even failure. Gaming also contributes to the development of a particular disposition well-suited to o an information-based culture and rapid change (New Media Consortium, 2011) 
El uso de los mundos virtuales en la educación es una herramienta ampliamente aceptada a nivel mundial (2011 Horizon Report ${ }^{3}$, SimTeach ${ }^{4}$, New Media

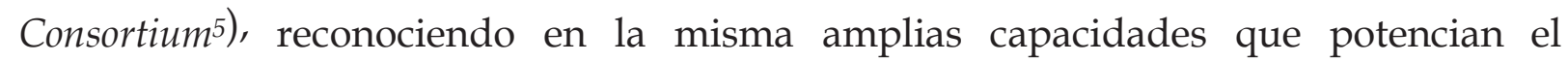
aprendizaje como la sensación de inmersión y presencia dentro del entorno tridimensional sumado a la posibilidad de experimentar, explorar y realizar simulaciones en tiempo real de forma conjunta con otros estudiantes compartiendo recursos online como videos, audio, textos o imágenes.

El sentido de "presencia" que ofrecen estas plataformas, a decir de Richard C. MacKinnon a partir del concepto cartesiano "Pienso, luego existo" en la cibersociedad es más adecuado afirmar "I am perceived, therefore I am" (MacKinnon,1994, p.119) el verse a uno mismo y a la vez ser visto por otros iguales en el metaverso, compartiendo un mismo lugar, nos transmite la señal visual de "estamos aquí juntos y en grupo", facilitando los grados de comunicación y la eficiencia en el desarrollo de diferentes actividades, en especial el aprendizaje de una forma más real.

Siguiendo en esta línea y basados en el concepto de edutainment, el uso de los Mundos Virtuales para adolescentes y jóvenes aplicados a e-learning resulta natural y accesible a los mismos, ya que permite vincular de manera efectiva el aprendizaje formal con el no formal del juego, acercándoles el conocimiento de una forma diferente, donde el aspecto lúdico y las formas de trabajo participativo dentro de un entorno tridimensional inmersivo les permiten no solo experimentar directamente sobre los objetos del conocimiento sino "hacer", realizar; lo que resulta esencial en situaciones de aprendizaje.

\section{METODOLOGÍA}

El estudio se basa tiene su base metodológica en el método análitico-sintético, el cual a partir del análisis y la síntesis, se descompone los mundos virtuales 3D o Metaversos en el ámbito de la educación en los diferentes tramos formativos, y simultaneámente a través de la síntesis, se integran estos vistos en su interrelación con la comunidad educativa. 


\section{ANÁLISIS Y DISCUSIÓN}

\subsection{Mundos virtuales 3D aplicados a la educación}

El movimiento hacia el mundo virtual como una enseñanza viable y entorno de aprendizaje parece imparable. (Kelton, 2008). A diferencia de los sistemas TIC tradicionales, la educación dentro de un entorno inmersivo introduce a niños, adolescentes y jóvenes al ambiente familiar de los videojuegos, acercándoles el conocimiento de una forma diferente, motivando el aprendizaje a través de actividades lúdicas como mini-juegos, lecciones interactivas y experimentos vivenciales, logrando de esta manera, mantener de manera continuada la atención de los estudiantes.

Aprender "haciendo" es considerada una de las formas más adecuadas para enseñar y aprender. Según Javier Martínez Aldanondo (2006), el hombre aprende de forma eficaz cuando el aprendizaje se convierte en un proceso de construcción activo de conocimiento e interacción entre pares y con otro más experimentado, realizado a través de la experiencia directa. De esta forma el alumno asume el proceso de aprendizaje como una experiencia propia.

La experiencia de aprendizaje dentro de los mundos virtuales, es una forma muy efectiva de enseñar cualquier tipo de proceso o procedimiento específico mediante simulaciones y juegos serios combinado con elementos más tradicionales como la lectura de textos, videos o conferencias sobre determinados temas en tiempo real y de forma inmersiva.

Los mundos virtuales 3D brindan la oportunidad de explorar nuevos métodos para la formación y el aprendizaje, proponiendo una nueva dimensión a la experiencia de alumnos y docentes en la experiencia del aprendizaje, ya que el diseño del propio entorno resulta motivador al sumar el aspecto lúdico al objeto mismo del conocimiento fomentando a la vez la confianza y el apoyo mutuo entre sus usuarios:

a) Mediante diferentes tipos de juegos y competiciones, así como otras actividades de aprendizaje social.

b) Mediante la organización de eventos y actividades que estimulen a los alumnos a integrarse con el entorno de aprendizaje, que posibiliten la interacción y el aprender unos de otros.

En este sentido los mundos virtuales 3D se constituye como una modalidad innovadora, puntera dentro de las TICs, que aprovecha todas las potencialidades que 
a) Permiten la interacción y la experimentación en tiempo real entre varias instituciones educativas localizadas en distintas ubicaciones geográficas.

b) Accesibilidad a todos los contenidos en el sentido más amplio de la palabra, ya que la presencia virtual favorece la integración de usuarios con discapacidades físicas o de movilidad.

c) El metaverso, al ser un contenedor online de contenidos audiovisuales posibilita la innovación en ideas creativas acordes a este nuevo medio, obteniendo por parte de los usuarios una rápida respuesta a los contenidos propuestos, con un coste menor en recursos que en la vida real.

d) Los mundos virtuales permiten expandir las posibilidades de captación de audiencia y ofertar contenidos educativos específicos para este nuevo modelo de formación.

\subsection{Experiencias educativas en los metaversos}

En la actualidad existen numerosas experiencias educativas a nivel mundial en variadas plataformas. A continuación se enumeran algunos ejemplos:

QuestAtlantis es un proyecto de Indiana University School of Education para la enseñanza de niños entre 9 y 16años realizado dentro de la plataforma Active Worlds. (Barab, S. et al, 2007)

a) Wilostars3D: 3D Virtual Worlds for K-12 Education es un mundo educativo para adolescentes.

b) Suffern Middle School es la primera escuela secundaria con presencia en el mundo virtual. La misma fue desarrollada dentro de la plataforma para adolecentes de Second Life llamada Teen Second Life. (Sheehy, P., 2008)

c) Olive Systems: Stanford Hospital para simulaciones médicas y entrenamiento.

d) Second Life: Entre las universidades más destacadas con presencia en este medio se encuentran el MIT, Harvard, Standford, Columbia, Princeton o Nueva York en EEUU, Frankfurt y Hamburgo en Alemania, Oxford, Edimburgo, Coventry, University of London, North Lanarkshire Council y Birmingham City University en el Reino Unido.

\subsection{Situación de la tecnología en España}


Los metaversos son en sí mismos entornos inmersivos 3D en tiempo real, cuya naturaleza de espacio contenedor los hace idóneos para alojar contenidos audiovisuales, surgiendo enormes posibilidades en el área formativa. Sin embargo, la falta de conocimiento y la inexperiencia en esta tecnología, impide a los diferentes actores que intervienen en los procesos educativos hacer uso de las mismas, y adentrarse de lleno es esta potente herramienta para explotarla en todas sus capacidades.

En el caso de España existen algunas experiencias fundamentalmente dentro del ámbito universitario llevadas adelante de forma experimental por grupos de profesores más que de manera corporativa. Tal es el caso de la Universidad de la Coruña, la Universitat Oberta de Catalunya, la Universidad Autónoma de Madrid, la Universidad Carlos III, la Universidad de Navarra, la Escuela Superior de Ingeniería de Vigo, la Universitat Rovira i Virgili, la Universidad Miguel Hernández, la Universidad a Distancia de Madrid o la Universidad de Granada.

Fuera del ámbito universitario existen experiencias formativas puntuales dentro de los mundos virtuales como "Comenius Virtual" 7desarrollado por IFO (Instituto de Formación Online) subvencionada dentro de la segunda convocatoria del Plan Avanza 2008.

A este respecto, los principales problemas y carencias detectadas en el ámbito del e- learning en relación con los mundos virtuales en el contexto español son:

a) No existe la experiencia necesaria para aprovechar las potencialidades de los mundos virtuales, perdiéndose de esta forma una potente herramienta para la formación.

b) No existe la experiencia necesaria en el desarrollo de espacios con contenidos audiovisuales formativos para niños y adolescentes dentro de los metaversos.

c) No existe un mecanismo de canalización de recursos de los mundos virtuales al mundo real ni viceversa. De esta forma se pierden los valores añadidos que enriquecerían la experiencia educativa.

Frente a todas las posibilidades que presentan estos entornos, se hace necesario investigar y desarrollar herramientas y contenidos más adecuados para llevar a cabo de manera más eficaz los espacios inmersivos para la educación preuniversitaria. 


\subsection{Mundos propietarios frente a mundos de código abierto}

En el caso de mundos inmersivos propietarios como Second Life una serie de servidores pertenecientes a las empresas propietarias controlan el acceso a la plataforma, la persistencia de mundos y regiones, el envío de los datos referentes a la simulación del mundo en el ordenador del usuario así como el almacenamiento de todas las configuraciones y datos asociados a los terrenos virtuales, a los avatares y a todos los contenidos creados por los mismos en tiempo real, guardados en el inventario o en el terreno.

Todos estos datos son almacenados en una base de datos en lenguajes interoperables como XML y en bases de datos estándares de facto como MySQL. Los servidores trabajan sincronizados para una efectiva conexión y desconexión al mundo por parte de los usuarios. El software cliente o Viewer instalado en la máquina del usuario le permite acceder a la plataforma e interactuar con el mundo.

Es importante hacer referencia a que todos los ejemplos de mundos virtuales mencionados anteriormente fueron realizados sobre plataformas de arquitectura propietaria de las respectivas empresas que llevan a cabo su explotación. Por tanto, las capacidades de los usuarios en términos de creación de contenidos y control sobre el mundo virtual están siempre supeditadas a la voluntad o fines comerciales de aquellas.

Sin embargo el uso de tecnología de código abierto salta esta limitación, tecnologías como OpenSim 8 , RealXtend ${ }^{9}$ o Open Wonderland ${ }^{10}$ permiten a los usuarios implementar sus propios servidores en los que alojar el mundo virtual, definir las capacidades de los mismos y tener, en suma, control total sobre el contenido que crean y la forma de explotarlo.

Analizando las diferentes plataformas se considera que las tecnologías de código abierto como OpenSim como la tecnología más adecuada donde desarrollar mundos aplicados a la educación considerando las siguientes ventajas en relación a las limitaciones de otras plataformas: 
a) Resulta una tecnología ideal para investigación y enseñanza, ya que es de código abierto, libre de usar, puede ser fácilmente modificado y ampliado, se puede instalar a nivel local con un costo mínimo posibilitando la posesión de un espacio virtual donde analizar, explorar y experimentar en metaversos.

b) La tecnología permite la interoperabilidad entre mundos, en especial con Second Life lo que posibilita que cualquier usuario del mismo acceda a OpenSim. Se espera que en breve sea posible que los avatares puedan teletransportarse directamente entre el entorno de Second Life y los distintos entornos privados de OpenSim lo que garantizará su popularidad.

c) Una arquitectura abierta, que permita tener una o varias islas en el disco duro de un servidor local conectadas a una red de mundos o grid global como OGP (Open Grid Public Beta) o a un grid privado

d) Terreno virtual de cualquier extensión, libre y gratuito.

e) Posibilidades de exportación/importación del inventario de Second Life utilizando herramientas como el software Second Inventory, donde todo el inventario de un usuario puede ser transferido de Second Life a OpenSim y viceversa.

f) Alternativas en el uso de visualizadores, pudiéndose elegir para acceder no solo el cliente oficial de Second Life, sino otras alternativas totalmente compatibles, con la capacidad para comunicarse indistintamente con la red de Second Life o con servidores OpenSim. Entre los diferentes visualizadores de código abierto disponible se encuentran Hippo Viewer, EyeCandy, Kirstens Viewer u Openlife Viewer.

g) Una gran comunidad de desarrolladores que colabora con OpenSim Project, lo que asegura su tecnología puntera, amplificando además sus posibilidades de aplicación a muy corto plazo.

\subsection{Experiencias dentro de plataformas abiertas}

Frente a todas las posibilidades que ofrecen plataformas como OpenSim se hizo factible su uso por parte de universidades que previamente habían utilizado Second Life. Un ejemplo de la migración de plataforma propietaria a una de código abierto son el Boston College, Future University Japan, Hong Kong Polytechnic o el Department of Computer Science and Technology de la Universidad de Bedfordshire.

Este último venía utilizando Second Life para la enseñanza de diversos proyectos de investigación desde 2007. Durante el año académico 2010/11 el equipo decidió migrar a ReactionGrid Inc. ${ }^{11}$ basada en OpenSim, con proyectos que incluyeron clases de programación LSL (Linden Scripting Lanquage) v clases sobre gestión de 
A nivel mundial proyectos como Open Cobalt Alpha ${ }^{12}$ o Pivote ${ }^{13}$ marcan una tendencia de lo que serán los espacios educativos 3D ya que la filosofía de código abierto de estas aplicaciones las convierten, por posibilidades y potencia, en competencia directa a los mundos virtuales propietarios.

En el caso de la enseñanza media reglada, Escocia ha sido de las pioneras en lograr incorporar los mundos virtuales de código abierto a su sistema educativo a través del proyecto CANVAS (Children's Art at the National Virtual Arena of Scotland), el cual es parte del programa educativo GLOW 13 , la comunidad online de escuelas escocesas. (Robertson, D., 2009).

Otras iniciativas de formación dentro de mundos de código abierto a nivel empresarial son las llevadas adelante por IBM, Raytheon, Siemens, Xerox o Microsoft con su Project Manhattan ${ }^{14}$ desarrollado por Microsoft Developer Community dentro de la plataforma OpenSim.

El proyecto se enfoca al trabajo colaborativo y la educación actuando como incubadora para desarrollos en tecnología de código abierto donde los usuarios pueden crear sus propias redes y simuladores. El proyecto tiene el apoyo de Microsoft, NET Developers User Group, TechNet Virtual User Group y G2.

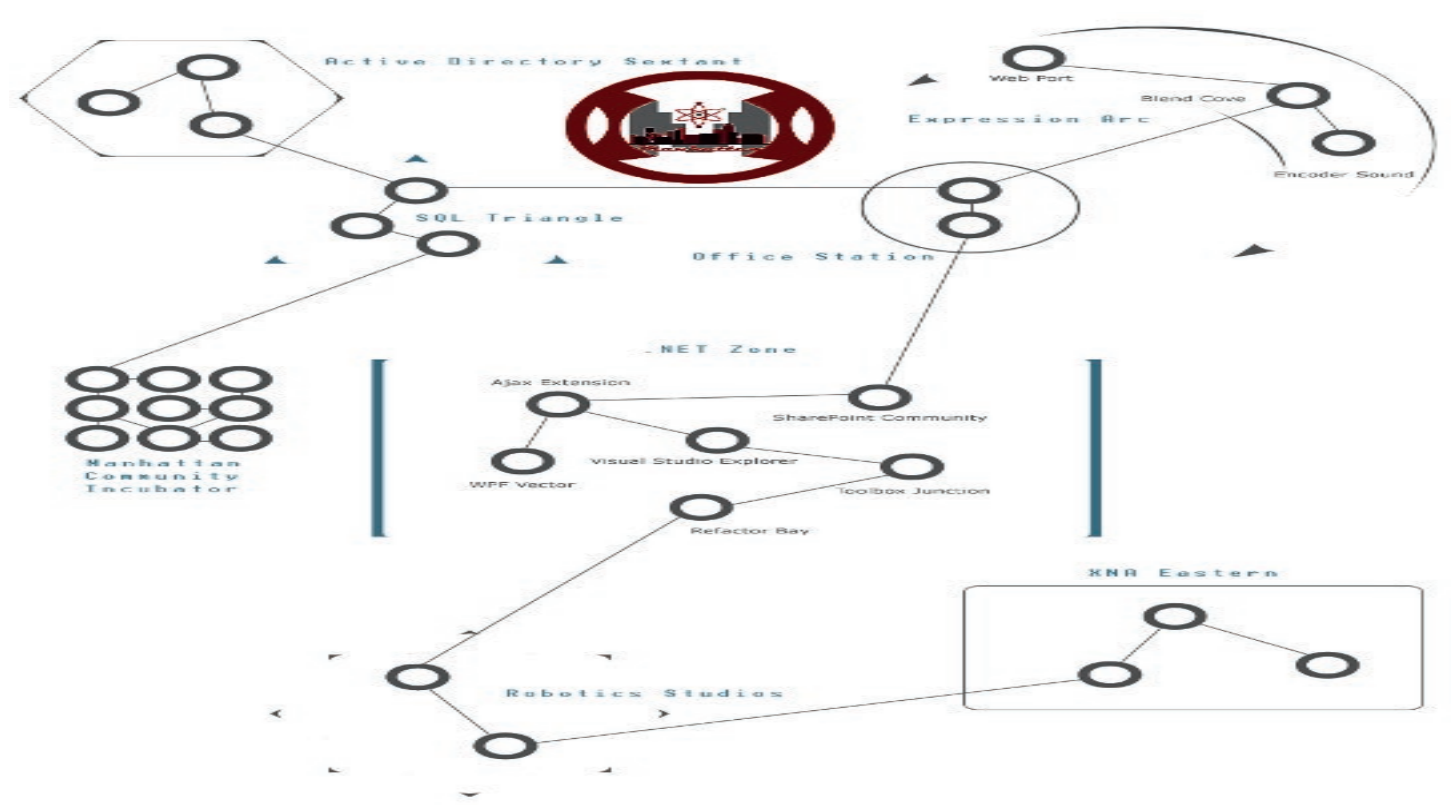

Figura 1. Organización de áreas en islas virtuales dentro del proyecto Manhattan 
Microsoft planteó su red de islas en el mundo virtual como laboratorio de investigación de aplicaciones de sus tecnologías (Office, SQL, .NET, etc.) dentro de los metaversos, contando también con zonas de incubación de negocio, áreas formativas, etc.

En paralelo, diferentes instituciones han desarrollado sus propios metaversos como la Universidad de Stanford y su mundo virtual Open Source denominado Sirikata ${ }^{15}$ o el proyecto Solipsis ${ }^{16}$ (Open Source Decentralized Metaverse), un metaverso abierto con tecnología puerto a puerto, donde el usuario crea sus propios contenidos desarrollado por France Telecom - Orange Group desde 2007 , dentro de la plataforma Media and Networks perteneciente a la plataforma europea NEM (Networked and Electronic Media).

\subsection{Casos de estudio desarrollados}

\section{Antecedentes: Isla Videa en Second Life}

Desde principios de 2008 la Universidad de A Coruña se sumó al frente de ola tecnológico que significaron en ese momento los mundos virtuales, con la apertura de un espacio virtual donde se desarrollaron dos cursos formativos dentro del programa del Master en Creación y Comunicación Digital.

Esta primera experiencia dentro de los metaversos brindó el "know-how" necesario para posteriormente investigar acerca de cuál es la tecnología más adecuada para trabajar en este tipo de proyectos.

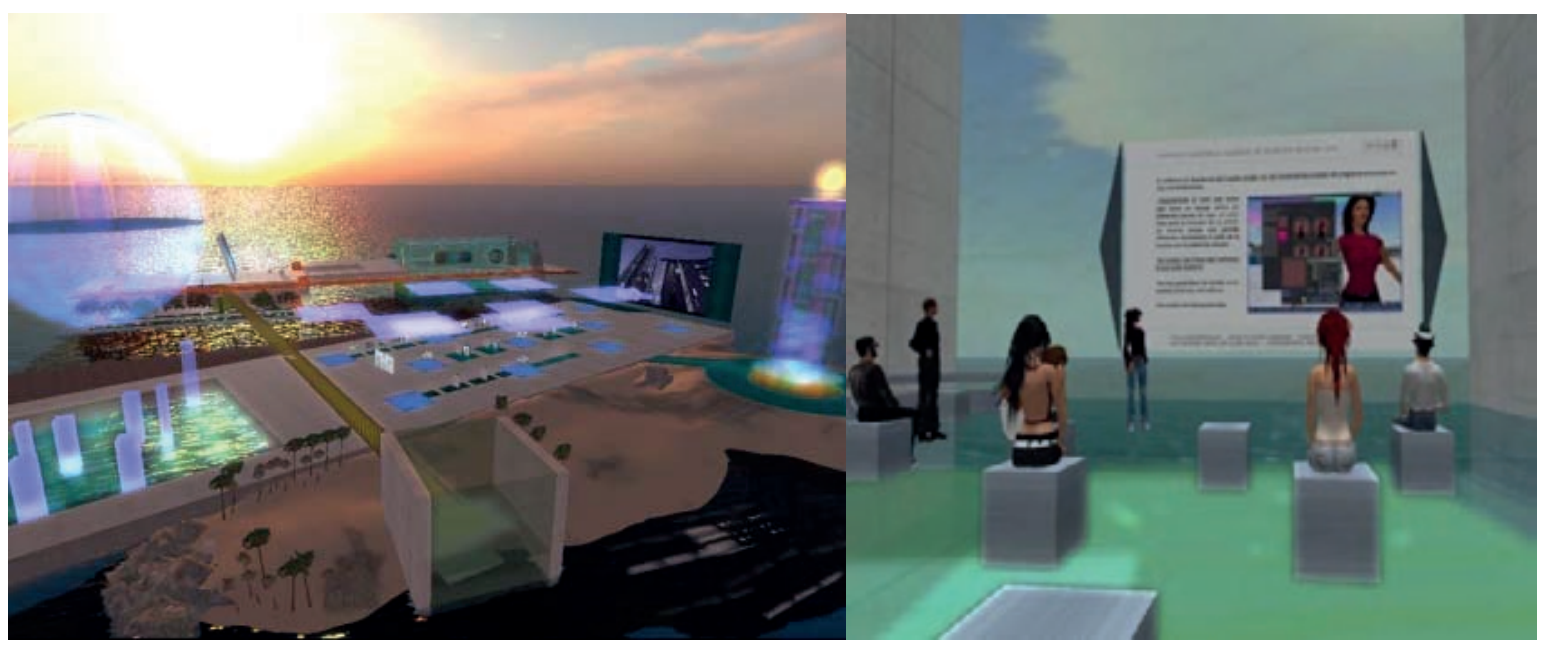

Figura 2. Isla Videa.

Figura.3 Anfiteatro 


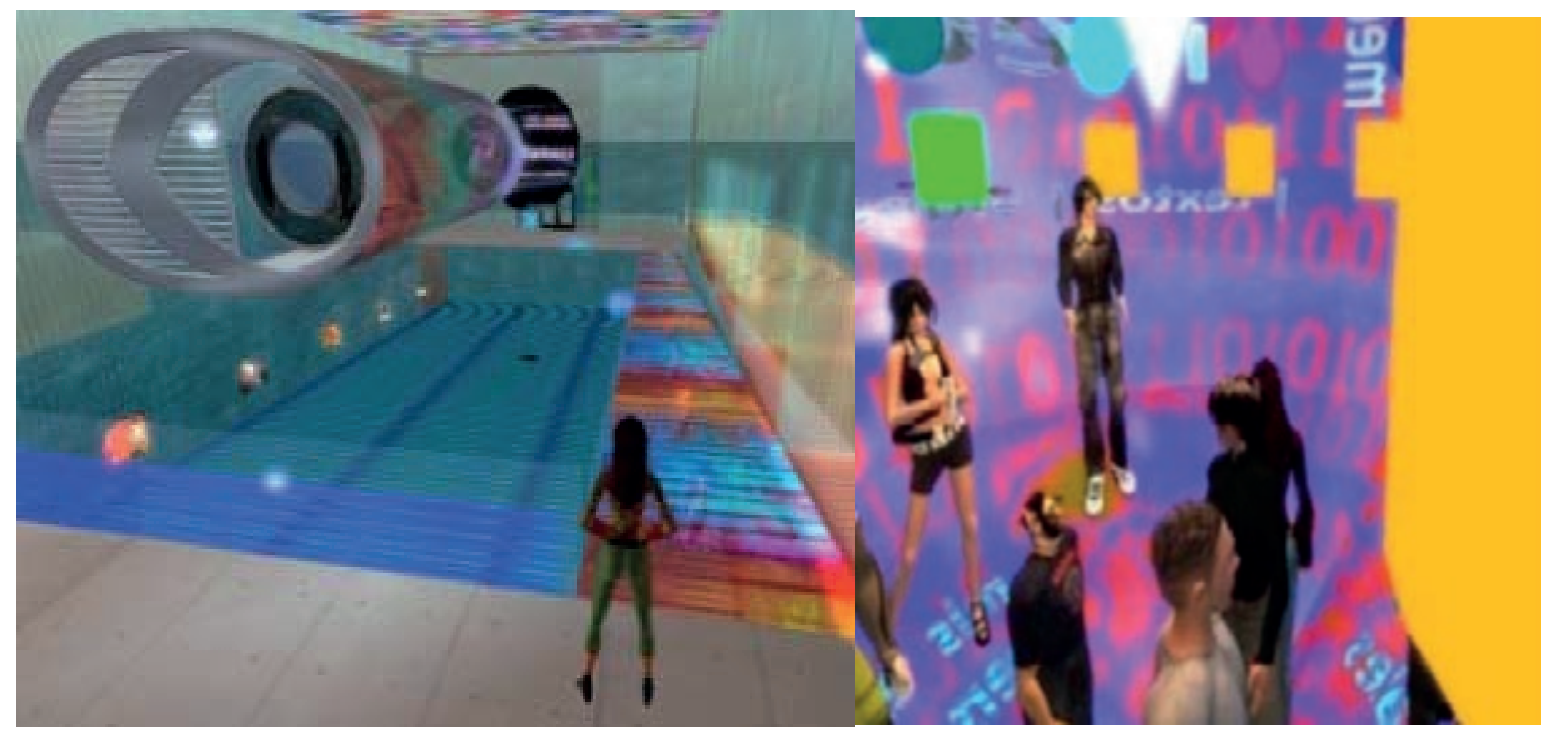

Figura.4 Aula flotante.

Figura. 5 Mediateca.

Fuente: Elaboración propia.

Desde el año 2008 los autores han estado investigando sobre tres plataformas de metaversos: Second Life17 OpenSim y Realxtend, realizándose pruebas en cada una de ellas, analizando comparativamente una serie de parámetros para definir el mundo virtual más acorde los objetivos prefijados en los diferentes proyectos.

Al finalizar el estudio se optó por la plataforma OpenSim considerando ventajas tales como la interoperabilidad entre mundos, en especial con Second Life; el coste cero del terreno virtual, la posibilidad de exportación/importación entre plataformas, y la existencia de una gran comunidad de desarrolladores que colabora con el denominado OpenSim Project, lo que asegura su tecnología actualizada.

En definitiva, el uso de software de código abierto resulta esencial en este tipo de proyectos tecnológicos para evitar adaptar las necesidades del proyecto a las limitaciones de un software propietario, depender del soporte de terceros, etc.

\section{Mundos educativos de código abierto}

Los mundos virtuales educativos a medida sobre plataforma de código abierto son, a nuestro criterio, una nueva y prometedora línea de investigación dentro del ámbito formativo. 
Mundo E-duka. (2010)

E-duKa es un mundo virtual educativo multiusuario, en 3D, a medida, sobre plataforma de código abierto dirigido a estudiantes y docentes de Enseñanza Secundaria Obligatoria. Este proyecto fue desarrollado para Netex Knowledge Factory S.L. Mundo E-duka.

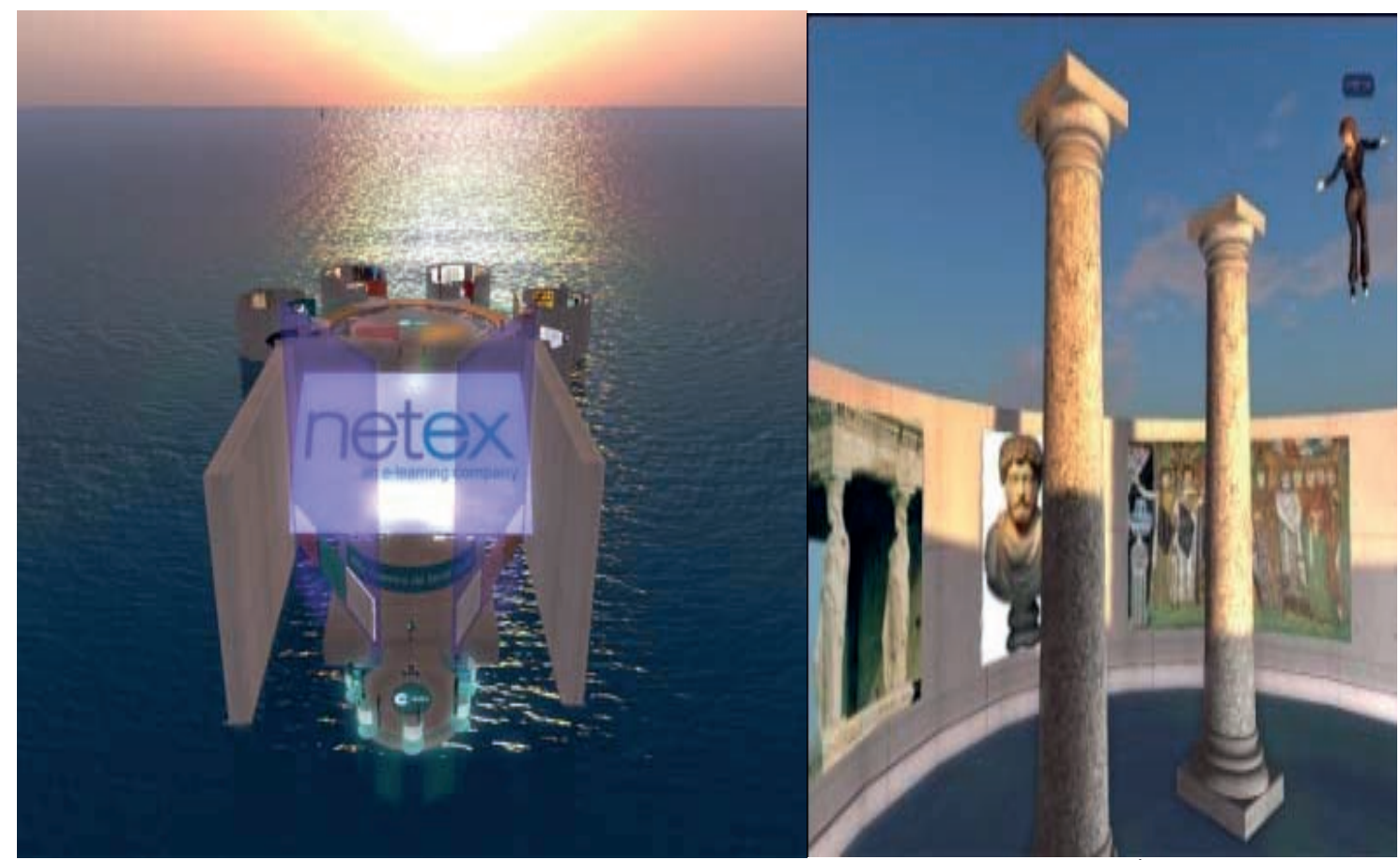

Figura 6. Vista general.

Figura 7. Área temática.

Fuente: Elaboración propia.

Mundo Agenda 21. (2011)

Mundo Agenda21 propone a creación de un mundo virtual 3D a medida que responde a las necesidades de varios ayuntamientos gallegos para acercar a la población de una manera novedosa y lúdica, herramientas educativas y expositivas sobre la sostenibilidad. Este proyecto fue desarrollado para Sustinere Alianza Estratégica. 


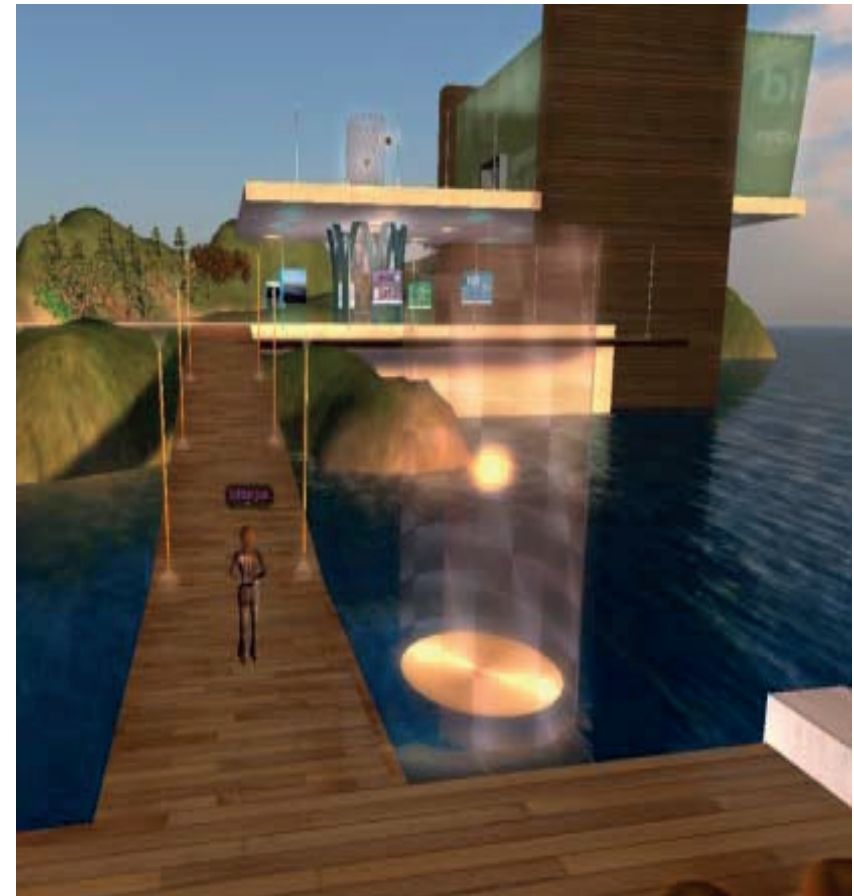

Figura 8. Vista general.

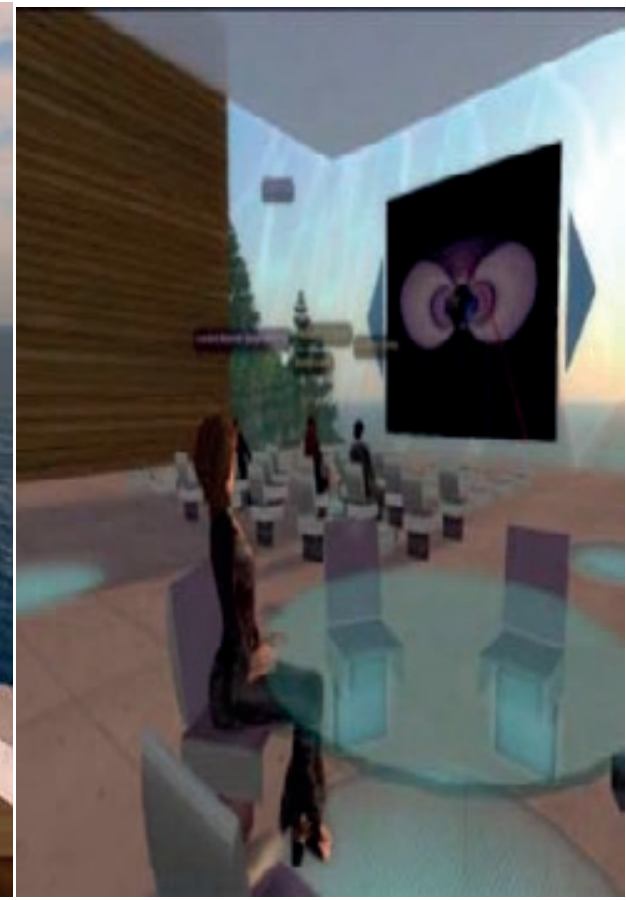

Figura 9. Clases.

Fuente: Elaboración propia.

Tanto en Mundo E-duKa y Mundo Agenda21 el uso de software de código abierto permite que el mismo se adapte a las necesidades del proyecto, aportando independencia, flexibilidad durante el proceso de desarrollo del producto final y asegurando un alto nivel de calidad del resultado; aprovechando el modelo de desarrollo colaborativo, no competitivo y de integración flexible de las aplicaciones de código abierto.

A todo lo anteriormente enumerado se suma la capacidad del lenguaje de script propio de estas plataformas, que define la interacción entre objetos y avatares, de forma que sus usuarios - a través de su avatar - realizan diferentes actividades como asistir a clases virtuales, compartir recursos multimedia (vídeos, aplicaciones Web, documentos) posibilitando el trabajo formativo de forma colaborativa entre varios usuarios de forma simultánea con un coste menor en recursos y dinero que en la vida real.

\subsection{Ciberarquitectura para entornos educativos}

Las nuevas formas de la arquitectura virtual dentro de los cibermundos, buscan responder a las nuevas necesidades del usuario contemporáneo, lo que permite

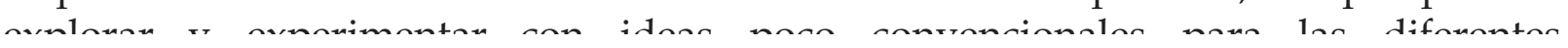


Las lógicas compositivas de la arquitectura de los metaversos surgen de las diferentes necesidades a dar respuesta, que son distintas de aquellas necesidades humanas básicas pero fundamentales para el Homo Sapiens, el Homo Faber y el Homo Ludens como el conocimiento y la exploración, la creación y el trabajo, la socialización y la recreación. (Barneche; Hernández, 2008).

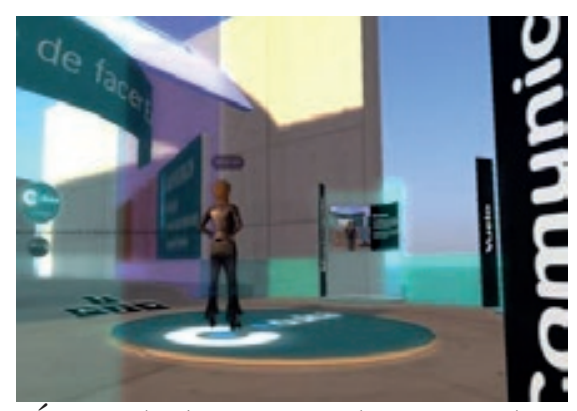

Figura 10. Área de bienvenida. Mundo Agenda21.

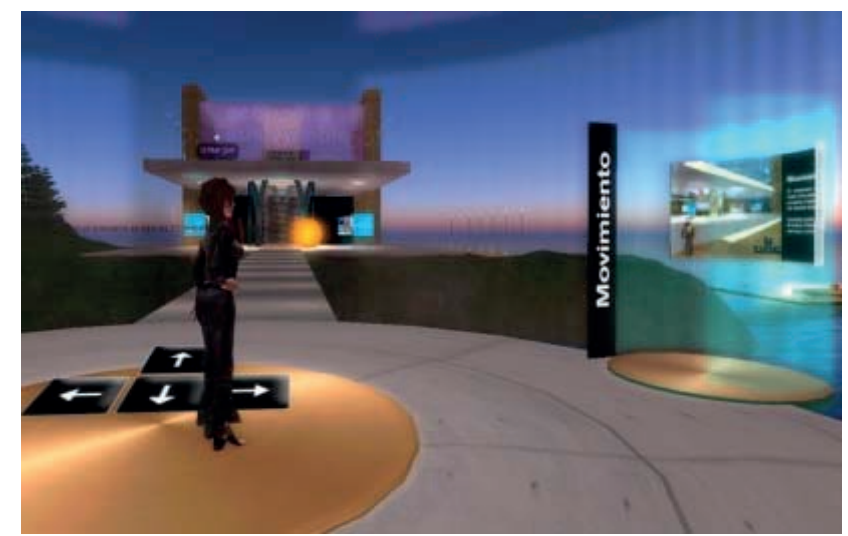

Figura 11. Mundo E-duka.

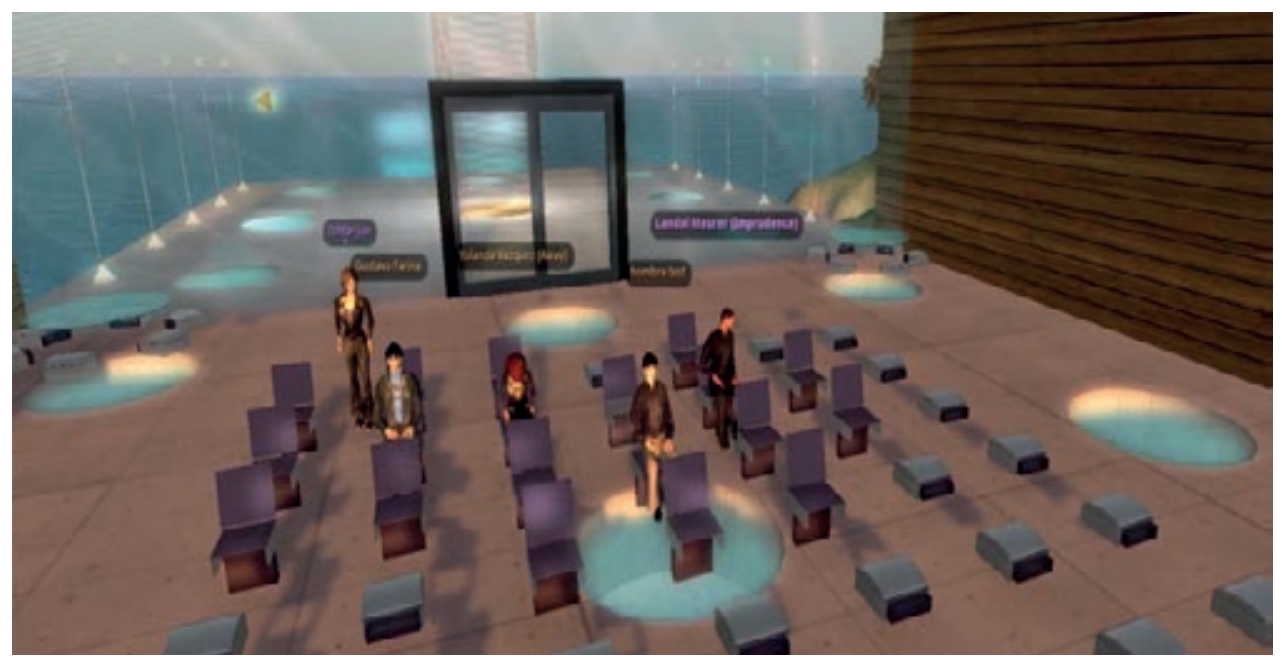


En este sentido, cada uno de los casos presentados fue diseñado para actuar como un entorno de aprendizaje flexible, con espacios que varían en función de las necesidades a responder, que conjuga aprendizaje y diversión, con objetos y contenidos totalmente interactivos de forma que resulten atractivos a los usuarios.

Paralelamente se proponen una serie de elementos complementarios con información textual, fotográfica y multimedia: video, sonidos, juegos y la propia Web.

Cada uno de los proyectos presentados consta de los siguientes espacios:

a) Un área de bienvenida: se trata de un espacio de acceso al mundo virtual, con comunicación al edificio central y a teletransportadores a los diferentes espacios virtuales. En esta zona se desarrolla el protocolo de entrenamiento en el uso del mundo en el primer acceso.

b) Un espacio central, compuesto por:

c) Área de exposición sobre temáticas de interés general.

d) Aulas de formación: Espacios para la enseñanza en grupo, mediante conferencias, con pantalla gigante para presentaciones de diapositivas, video y audio procedente del mundo real.

En el caso de Mundo E-duka se plantearon una serie de espacios temáticos de las diferentes materias formativas relacionadas con las asignaturas impartidas en la Enseñanza Secundaria Obligatoria.
a) Matemáticas
b) Lengua y Literatura
c) Geografía e Historia
d) Cultura clásica.
e) Ciencias de la Naturaleza
f) Tecnologías
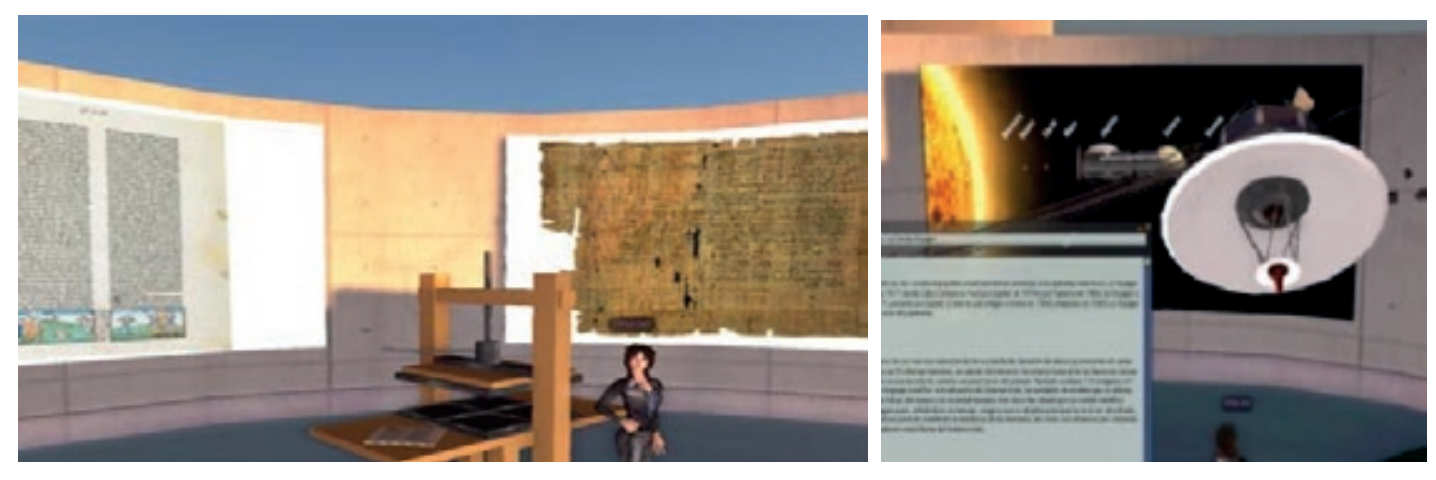
Cada uno de estos espacios está dotado de dispositivos de reproducción de material multimedia, así como contenidos tridimensionales interactivos relacionados con cada una de las temáticas.

El estudiante se acerca así de forma inmersiva e interactiva a los diferentes aspectos de sus áreas de interés; por ejemplo, en el caso de Mundo E-duka, el alumno puede conocer el Universo, la Vía Láctea y nuestro planeta, visualizando un movimiento de rotación completo. También puede saber sobre las diferentes especies que habitan el planeta, conocer cómo funciona una imprenta o como vivía un legionario romano.

\section{CONCLUSIONES}

Existen múltiples iniciativas en todo el mundo de aplicación de la tecnología de metaversos en educación, lo cual prueba su aplicabilidad en la docencia en múltiples campos y para todos los niveles formativos.

La creación de espacios virtuales para alojar las actividades formativas debe seguir criterios de diseño análogos en cuanto a rigor y calidad que el diseño de espacios fomativos para el mundo real. Su organización y definición formal sigue las reglas de diseño arquitectónico real, matizadas con las características únicas del metaverso.

\section{REFERENCIAS}

Alhadeff, E. (2011). Gamification: the path for innovative organizations emulates Serious Games. Recuperado el diciembre de 2011, de http:/ / seriousgamesmarket.blogspot.com/2011/04/gamification-path-forinnovative.html.

Álvarez, A. (2009). 12 tendencias tecnológicas que marcarán los próximos 5 años (20092013). Telefónica I+D. La Cofa, Blog de Vigilancia Tecnológica. Recuperado el diciembre de 2011, de www.lacofa.es/index.php /tecnologias/12-tendencias-tecnologicas-quemarcaran-los- proximos-5-anos-2009-2013.

Armstrong, R. (2009). Millions and millions of little monsters. Recuperado el diciembre de 2011, de www.independent.co.uk/life-style7gadgets-and-tech/gaming/millionsand-millions- of-little-monsters-1833531.html.

Barab, S.; Gresalfi, M.; Arici, A. (2009). Why educators should care about Games. Education Leadership Teaching for the 21st Century, 67. Recuperado el 2 de diciembre de 
Barab, S.; Dodge, T.; Thomas, M.; Jackson, C. \& Tuzun, H. (2007). Our Designs and the Social Agendas They Carry. The Journal of the Learning Sciences, 16: 263-305. Recuperado el 2 de diciembre de 2011, de http:/ / atlantis.crlt.indiana.edu / public/publications/critical_design_59.pdf.

Barab, S. (2011). Atlantis Remixed and Quest Atlantis. Recuperado el 3 de diciembre de 2011, de http:/ / atlantisremixed.org/.

Barneche, V. \& Hernández, L. (2008). Ciberarquitectura Educativa. La experiencia de Isla Videa en Second Life. En XII Congreso Iberoamericano de Gráfica Digital. La Habana: Ed Cujae.

Conrad, M.(2011). Leaving the Lindens: Teaching in Virtual Worlds of Other Provider. Recuperado el 2 de diciembre de 2011, de http://sl.perisic.com/relive11/MarcConrad LeavingTheLindens Final.pdf.

GARTNER Inc. (2011). Hype Cycle Special Report. Recuperado el diciembre de 2011, de www.gartner.com/it/page.jsp?id=1763814.

Kelton, A.J. (2008). Virtual Worlds? Outlook Good. Educause Review, 43(5). Recuperado el 5 de diciembre de 2011, de www.educause.edu/EDUCAUSE+Review / EDUCAUSEReviewMagazineVolume43/VirtualWorldsOutlookGood/163161.

Kzero Worldswide Consulting Co. (2011). Age profile for Virtual Worlds user bases. Recuperado el 6 de agosto de 2011, de www.kzero.co.uk/blog/.

Mackinnon, R. C. (1995). Searching for the Leviathan in Usenet. California: Editorial Steven G. Jones. Thousand Oaks

Martínez Aldanondo, J. (2006). Elearning en Blanco y Negro. Recuperado el 3 de diciembre de 2011, de http://www.learningreview.es/e-learning-279/informestemas-200/360- elearning-en-blanco-y-negro.

New media consortium. (2011). The NMC Horizon Report: 2011 K-12 Edition. Recuperado el 4 de diciembre de 2011, de www.nmc.org/pdf/2011-Horizon-ReportK12.pdf.

Robertson, D. (2009). CANVAS: Scotland's first schools based virtual world for learning. Recuperado el 8 de diciembre de 2011 de, http:/ /ltsblogs.org.uk/consolarium/2009/08/25/canvas- scotlands-first-schoolsbased-virtual-world-for-learning/. 
Wilostar 3D L.L.C. (2011). Online Homeschooling and 3D Virtual Worlds for K-12 Education. Recuperado el 7 de diciembre de 2011, de http:/ / www.wilostar3d.com.

\section{Viviana Barneche Naya}

Arquitecta, Universidad de la República, Uruguay. PhD candidate, en etapa de Tesis, Universidad de A Coruña. Docente de Taller de Anteproyectos, Facultad de Arquitectura, UdelaR (2001-2011). Docente dentro del Máster en Creación y Comunicación Digital, Universidad de A Coruña (2008-2009). Profesora interina, Universidad de A Coruña (2011). Sus líneas de investigación se enmarcan en los campos de la creación digital interactiva, el diseño de ciberarquitectura y contenidos dentro de los Metaversos, la recreación patrimonial, la simulación por computador y las Interfaces Naturales.

\section{Rocío Mihura López}

Licenciada en Bellas Artes por la Universidade de Vigo. Máster en Creación y Comunicación Digital por la Universidade da Coruña. PhD candidate, en etapa de Tesis, Universidad de A Coruña. Profesora en la Facultade de Ciencias da Comunicación de la Universidade da Coruña. Sus líneas de investigación abarcan los campos de la creación digital interactiva en el ámbito de la museística y los contenidos audiovisuales dentro de los Museos.

\section{Luis Antonio Hernández Ibáñez}

Doctor Arquitecto, Universidad de A Coruña, España. Director del Grupo de Visualización Avanzada en Arquitectura, Ingeniería Civil y Urbanismo - VideaLAB, Director del Máster en Creación y Comunicación Digital. (1998-2009). Profesor Titular en la Escuela Técnica Superior de Ingenieros de Caminos, Canales y Puertos; y de la Facultad de Ciencias de la Comunicación de la Universidad de A Coruña. Autor de múltiples publicaciones en el ámbito de la visualización avanzada, la ciberarquitectura y la recreación del Patrimonio. Sus líneas de investigación en enmarcan en el campo de la creación digital, la simulación por computador, las Interfaces Naturales, los Mundos Virtuales 3D y la Realidad Virtual. Miembro de IEEE, ACM Siggraph, Sigradi y SEAV. 